\title{
What can we learn from femtoscopic and angular correlations of identified particles in ALICE?
}

\author{
Łukasz Kamil Graczykowski ${ }^{1, \star}$ \\ for the ALICE Collaboration \\ ${ }^{1}$ Faculty of Physics, Warsaw University of Technology \\ Koszykowa 75, 00-662 Warszawa, Poland
}

\begin{abstract}
Two-particle correlations have proven to be a robust tool which allow the exploration of many physics phenomena present in high-energy particle collisions. In this work, two techniques, namely femtoscopic and angular correlations of identified particles, are discussed the most recent results of both of them obtained from $\mathrm{pp}$ and $\mathrm{Pb}-\mathrm{Pb}$ collisions recorded by the ALICE detector are presented.
\end{abstract}

\section{Introduction}

ALICE (A Large Ion Collider Experiment) [1] is one of the four large detectors located at the LHC (Large Hadron Collider) facility [2] at CERN - the European Organization for Nuclear Research. The detector was specifically designed to investigate ultra-relativistic $\mathrm{Pb}-\mathrm{Pb}$ collisions, where the very high temperatures and energy densities allow for the production of the Quark-Gluon Plasma (QGP) [3-7]. In addition, the ALICE detector has an extensive physics program analyzing protonproton and proton-lead collisions which are also delivered by the LHC.

The ALICE detector has some unique features. Among the most important is excellent tracking and particle identification (PID) over a broad momentum range which makes a variety of different measurements possible, not only connected to the studies of the QGP. Two-particle correlation measurements, namely femtoscopy and angular correlations, have been performed for different particle pairs and allowed for calculations of previously poorly known pair cross sections and shed new light on particle fragmentation models. Both techniques reported in this paper use the same definition of correlation function, expressed as a ratio of correlated pairs from the same event to the reference distribution containing pairs of particles coming from different events. The correlation functions are normalized in the way that the reported distributions represent the ratio of probabilities.

\section{Femtoscopy}

Femtoscopy is the term describing two-particle correlations measured as a function of relative momentum $q=2 \cdot k^{*}=p_{1}-p_{2}$ which are used to gather information about the spatio-temporal properties

^e-mail: lukasz.graczykowski@pw.edu.pl 
of the collision. The femtoscopic correlation is related to the source and the final-state interaction via the Koonin-Pratt equation $[8,9]$

$$
C\left(k^{*}\right)=\int S\left(\vec{r}^{*}\right)\left|\Psi_{-\vec{k}^{*}}\left(\vec{r}^{*}\right)\right| d^{3} \vec{r}^{*}
$$

where $S\left(\vec{r}^{*}\right)$ is the source function, typically a one-dimensional Gaussian $S\left(\vec{r}^{*}\right) \sim e^{-\left|\vec{r}^{*}\right|^{2} /\left(4 R^{2}\right)}$ (where $R$ is the width corresponding to the source size, sometimes referred to as the "HBT radius"), $\left|\vec{r}^{*}\right|$ is the relative distance in the pair-rest frame (PRF) and $\Psi_{-\vec{k}^{*}}\left(\vec{r}^{*}\right)$ is the two-particle interaction kernel (solution to the scattering problem). Generally, $\Psi_{-\vec{k}^{*}}\left(\vec{r}^{*}\right)$ may include effects arising due to the effects of quantum statistics and final-state interactions, depending on the studied pair.

Traditionally, in femtoscopy the form of $\Psi_{-\vec{k}^{*}}\left(\vec{r}^{*}\right)$ is known and the parameters of $S\left(\vec{r}^{*}\right)$, which describe the shape and evolution of the source, are extracted. In fact, Eq. (1) allows us to utilize femtoscopic technique in a novel way, constraining $S\left(\vec{r}^{*}\right)$ from other measurements and extracting information on $\Psi_{-\vec{k}^{*}}\left(\vec{r}^{*}\right)$. In particular, if only the strong interaction is present, $\Psi_{-\vec{k}^{*}}\left(\vec{r}^{*}\right)$ has the following form of a superposition of a plane wave and a spherical wave

$$
\Psi_{-\vec{k}^{*}}\left(\vec{r}^{*}\right)=e^{-i \vec{k}^{*} \cdot \vec{r}^{*}}+f\left(k^{*}\right) \frac{e^{i k^{*} \cdot r^{*}}}{r^{*}},
$$

where $f\left(k^{*}\right)$ is the $s$-wave scattering amplitude which has a different form depending on the particle pair under study. Analytical solutions of Eq. (1) with $\Psi_{-\vec{k}^{*}}\left(\vec{r}^{*}\right)$ accounting for the strong interaction, in the effective range approximation, were introduced for different pair systems by R. Lednicky [10, 11].

This paper focuses on two such measurements are presented. Firstly, correlations of baryons with anti-baryons are discussed in Sec. 2.1 and secondly, correlations between neutral and charged kaons are presented in Sec. 2.2.

\subsection{Baryon-anti-baryon pairs}

One of the most important problems in QCD is to understand the interaction between two baryons. While significant amount of experimental data exists for baryon-baryon pairs [12], no measurements exist for baryon-anti-baryon systems other than $\mathrm{p} \overline{\mathrm{p}}, \mathrm{p} \overline{\mathrm{d}}$, and $\mathrm{p} \overline{\mathrm{n}}$. UrQMD, a current re-scattering model widely used in the field of collisions of heavy ions, assumes baryon-anti-baryon interaction from scaling of $\mathrm{p} \overline{\mathrm{p}}$ interaction parameters on the basis of the Additive Quark Model [13].

In general, femtoscopy can be used to extract the interaction parameters of such systems. The scattering amplitude $f\left(k^{*}\right)$ in Eq. (2) in this scenario becomes (in the effective range approximation)

$$
f\left(k^{*}\right)=\left[\frac{1}{f_{0}}+\frac{1}{2} d_{0} k^{* 2}-i k^{*}\right]^{-1},
$$

where $f_{0}$ is the scattering length and $d_{0}$ is the effective range of the interaction. An attempt to experimentally constrain the parameters of $\mathrm{p} \bar{\Lambda}$ pairs using femtoscopy was made by the STAR experiment at RHIC [14]. In particular, both real and imaginary parts of $f_{0}$ were extracted manifesting the presence of a significant non-elastic channel, annihilation, while the extracted source size ("HBT radius") was significantly lower than the results from baryon-baryon pairs at similar transverse mass $m_{\mathrm{T}}$. These results seemed to be in contradiction to hydrodynamic model calculations which predict $1 / m_{\mathrm{T}}$ scaling which has been confirmed by RHIC and LHC results of meson and baryon correlations. This inconsistency was studied later in Ref. [15] which concludes that the so-called residual correlations ${ }^{1}$ were

\footnotetext{
${ }^{1}$ Correlations arising from admixture in the sample of particles of the same type being products of heavier baryon decays.
} 
not properly included in the original study. The results were re-analyzed with the proper accounting for those correlations.

In this paper, the recent ALICE measurements extending the femtoscopic studies of baryon-antibaryon interactions on $\Lambda \bar{\Lambda}$ pairs are discussed. In addition, a procedure to fit simultaneously all measured baryon-anti-baryon systems is introduced. The analysis was carried out for $\mathrm{p} \overline{\mathrm{p}}, \mathrm{p} \bar{\Lambda}(\overline{\mathrm{p}} \Lambda)$, and $\Lambda \bar{\Lambda}$ pairs on $\mathrm{Pb}-\mathrm{Pb}$ collisions at the LHC recorded at the center-of-mass energies of $\sqrt{s_{\mathrm{NN}}}=$ $2.76 \mathrm{TeV}$ and $\sqrt{s_{\mathrm{NN}}}=5.02 \mathrm{TeV}$, respectively. In addition, six centrality ranges were introduced, giving in total 36 measured baryon-anti-baryon correlation functions. The $\chi^{2}$ was calculated globally, representing the minimum of the fit to all functions together. For each pair a proper form of correlation function was chosen, taking into account the strong interaction as well as Coulomb repulsion in case of the $\mathrm{p} \overline{\mathrm{p}}$ system. Residual correlations were also taken into account with the fractions of residual pairs estimated from the AMPT model [16] and decay kinematics from the THERMINATOR 2 model [17]. The parameters of the interaction of residual pairs are the same as those for experimentally measured pairs, and are free parameters of the fit. In addition, the scaling of "HBT radii" with $m_{\mathrm{T}}$ and the cube root of multiplicity (relating to different centralities) was assumed in order to constrain the radius. Finally, three sets of interaction parameters were introduced, namely for $\mathrm{p} \bar{\Lambda}(\overline{\mathrm{p}} \Lambda)$ and $\Lambda \bar{\Lambda}$ pairs as well as parameters of an effective potential for other baryon-anti-baryon pairs not measured directly but present as residual correlation contributions.

Figure 1 presents baryon-anti-baryon femtoscopic correlation functions measured in $\mathrm{Pb}-\mathrm{Pb}$ collisions at both studied collision energies. The Lednicky fit shows a good description of experimental data. The scattering parameters - the results of the fit, compared to measurements from other experiments and model predictions, are shown in Fig. 2.
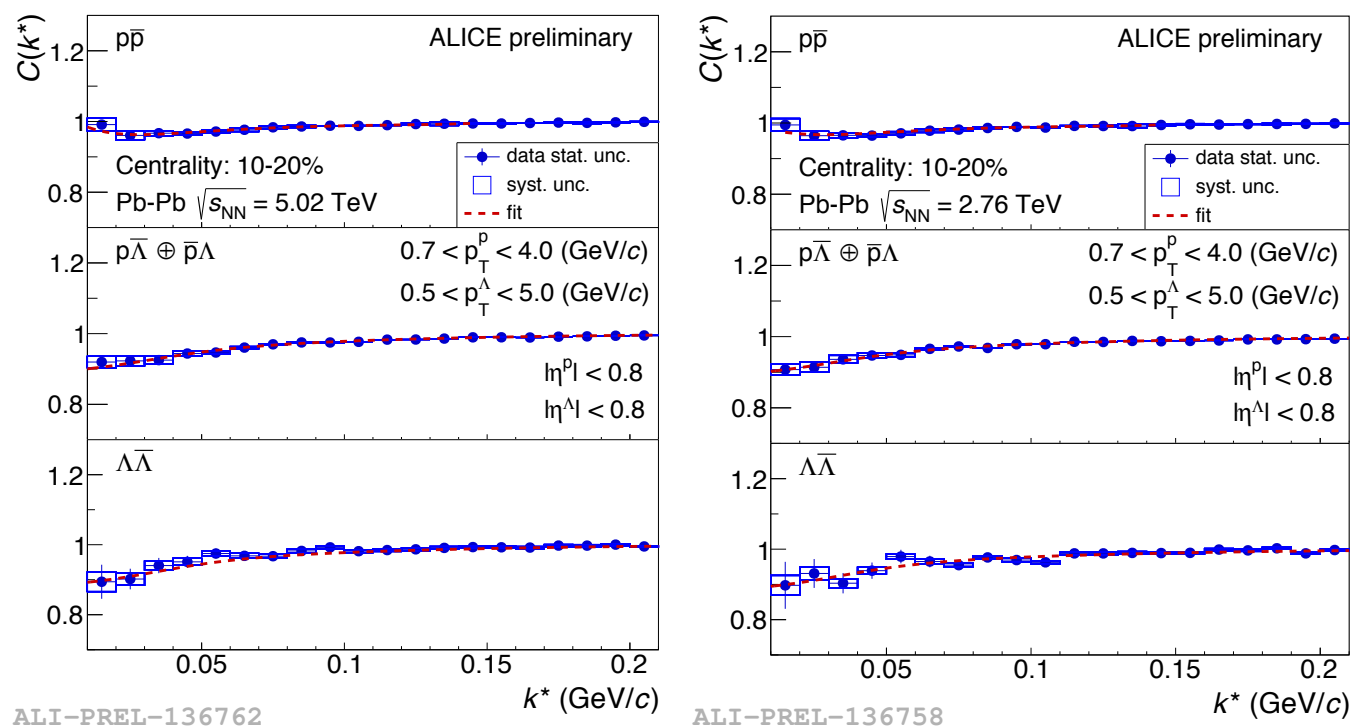

Figure 1. Femtoscopic correlation functions for $\mathrm{p} \overline{\mathrm{p}}, \mathrm{p} \bar{\Lambda}+\overline{\mathrm{p}} \Lambda$, and $\Lambda \bar{\Lambda}$ pairs from $\mathrm{Pb}-\mathrm{Pb}$ collisions at $\sqrt{s_{\mathrm{NN}}}=$ $5.02 \mathrm{TeV}$ (left) and $\sqrt{s_{\mathrm{NN}}}=2.76 \mathrm{TeV}$ (right). Red lines represent Lednicky fits.

There are a few conclusions that can be drawn from these results: (I) All the extracted scattering parameters are similar to each other meaning that the interaction for different baryon-anti-baryon 

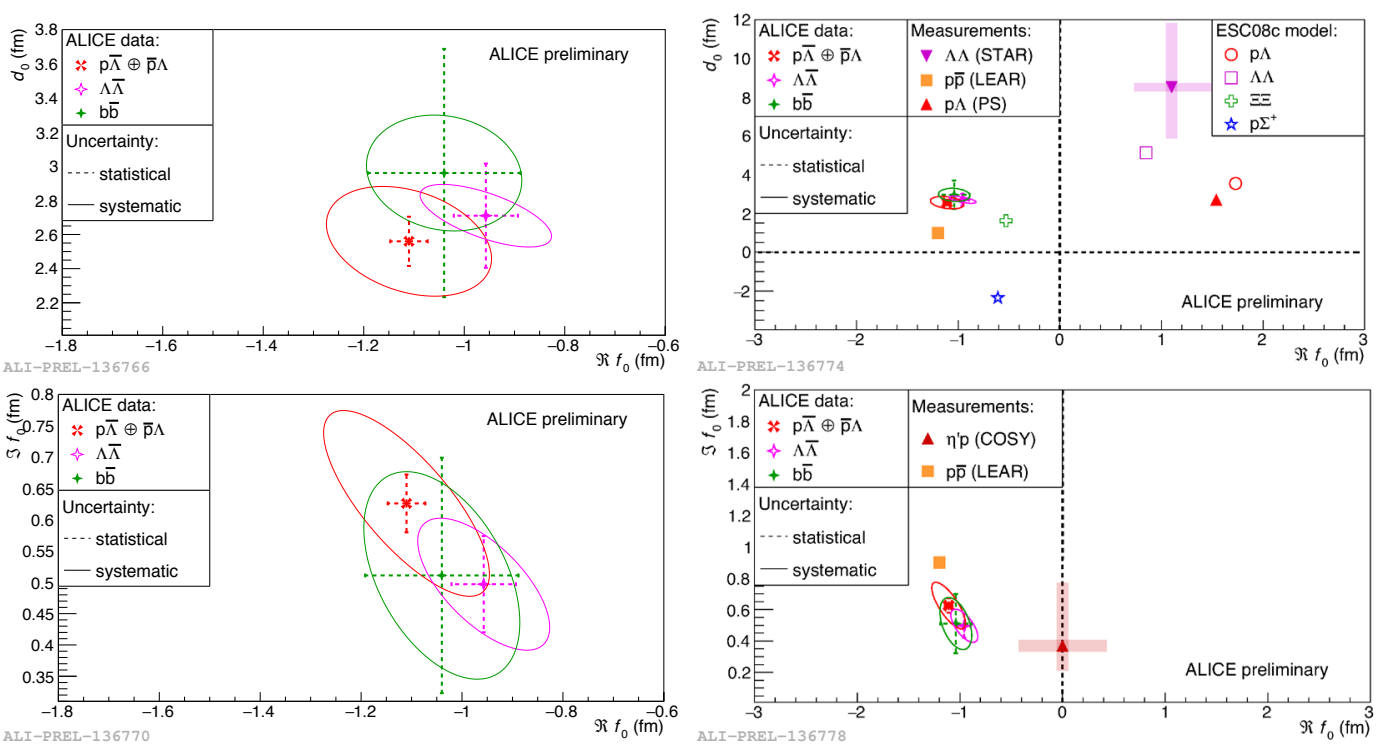

Figure 2. (Left) Scattering parameters $f_{0}$ and $d_{0}$ for $\mathrm{p} \bar{\Lambda}+\overline{\mathrm{p}} \Lambda, \Lambda \bar{\Lambda}$ pairs and effective scattering parameters describing other baryon-anti-baryon pairs. (Right) Comparison to other measurements and model predictions.

pairs is comparable. (II) The real part of the scattering length $\mathfrak{R} f_{0}$ is negative which suggests either the existence of baryon-anti-baryon bound states or a repulsive strong interaction. (III) The imaginary part of the scattering length $\mathfrak{J} f_{0}$ is positive, reflecting the presence of a significant inelastic channel (in this case baryon-anti-baryon annihilation). (IV) Finally, the effective range $d_{0}$ is not zero which suggests that the assumptions made in previous analyses, where it was fixed to zero in order to reduce the number of free parameters, are not justified $[14,15]$.

\subsection{Neutral kaon-charged kaon pairs}

The first measurements of $\mathrm{K}_{\mathrm{S}}^{0} \mathrm{~K}^{ \pm}$femtoscopic correlations were performed by ALICE in $\mathrm{Pb}-\mathrm{Pb}$ collisions at $\sqrt{s_{\mathrm{NN}}}=5.02 \mathrm{TeV}$ [18]. In addition, the complementary preliminary results in $\sqrt{s}=7 \mathrm{TeV}$ pp collisions from ALICE have also recently become available. The only allowed process (source of correlation) which contributes to the femtoscopic correlation function for these particle pairs is the strong final-state interaction. It proceeds through the $\mathrm{a}_{0}(980)$ near-threshold resonance with the scattering amplitude $f\left(k^{*}\right)$ from Eq. (2) of the form:

$$
f\left(k^{*}\right)=\frac{\gamma_{\mathrm{a}_{0} \rightarrow \mathrm{K}} \overline{\mathrm{K}}}{m_{\mathrm{a}_{0}}^{2}-s-i\left(\gamma_{\mathrm{a}_{0} \rightarrow \mathrm{K}} k^{*}+\gamma_{\mathrm{a}_{0} \rightarrow \pi \eta} k_{\pi \eta}\right)},
$$

where $m_{\mathrm{a}_{0}}$ is the mass of the $\mathrm{a}_{0}$ resonance, $\gamma_{\mathrm{a}_{0} \rightarrow \mathrm{KK}}$ and $\gamma_{\mathrm{a}_{0} \rightarrow \pi \eta}$ are the $\mathrm{a}_{0}$ coupling parameters of the $\mathrm{a}_{0}$ resonance to the $\mathrm{K}_{\mathrm{S}}^{0} \mathrm{~K}^{-}$(or $\overline{\mathrm{K}}^{0} \mathrm{~K}^{+}$) and $\pi \eta$ channels, respectively. The $k_{\pi \eta}$ is the momentum in the $\mathrm{a}_{0} \rightarrow \pi \eta$ channel while $s$ is defined as $s=4\left(m_{\mathrm{K}^{0}}^{2}+k^{* 2}\right)$. In this analysis several sets of coupling parameters of $\mathrm{a}_{0}$ (in the order of lower to higher values) taken from [19] ("Martin"), [20] (“Antonelli"), [21] (“Achasov1"), and [22] (“Achasov2") were studied. 
The main motivation for studying the $\mathrm{K}_{\mathrm{S}}^{0} \mathrm{~K}^{ \pm}$final-state interaction is the possibility of constraining the coupling parameters of $\mathrm{a}_{0}$ which in fact is considered in the literature as a potential tetraquark state, a hadron composed of 4 constituent quarks, or a " $\bar{K}-$ K molecule" [19-24].

The advantage of ALICE is the possibility of performing the same analysis on both collision systems, where different underlying physics phenomena are present. This allows to cross-check the two results and better constrain the extracted $a_{0}$ parameters. The tetraquark hypothesis could also be supported if the measurements lead to the same results.

Figures 3 and 4 show femtoscopic correlation functions (corrected for min-jet underlying correlations) of $\mathrm{K}_{\mathrm{S}}^{0} \mathrm{~K}^{ \pm}$pairs measured in $\mathrm{Pb}-\mathrm{Pb}$ and $\mathrm{pp}$ collision systems, respectively, in different pair transverse momentum $k_{\mathrm{T}}=\left|p_{\mathrm{T}, 1}+p_{\mathrm{T}, 2}\right| / 2$ ranges. Blue points represent the experimental data while the red lines correspond to the fit function. One can clearly see from both figures that the $\mathrm{a}_{0}$ parameterization of the femtoscopic signal describes very precisely the experimental result. The extracted source sizes $R$ are compared to the results of identical kaon femtoscopy from ALICE [25, 26], which is shown in Fig. 5. The "Martin" parameterization [19], which contains the lowest values of $a_{0}$ coupling parameters, gives radii which lie significantly below those of identical kaon femtoscopy. Therefore, higher values of $\mathrm{a}_{0}$ resonance are favored by the experimental data.

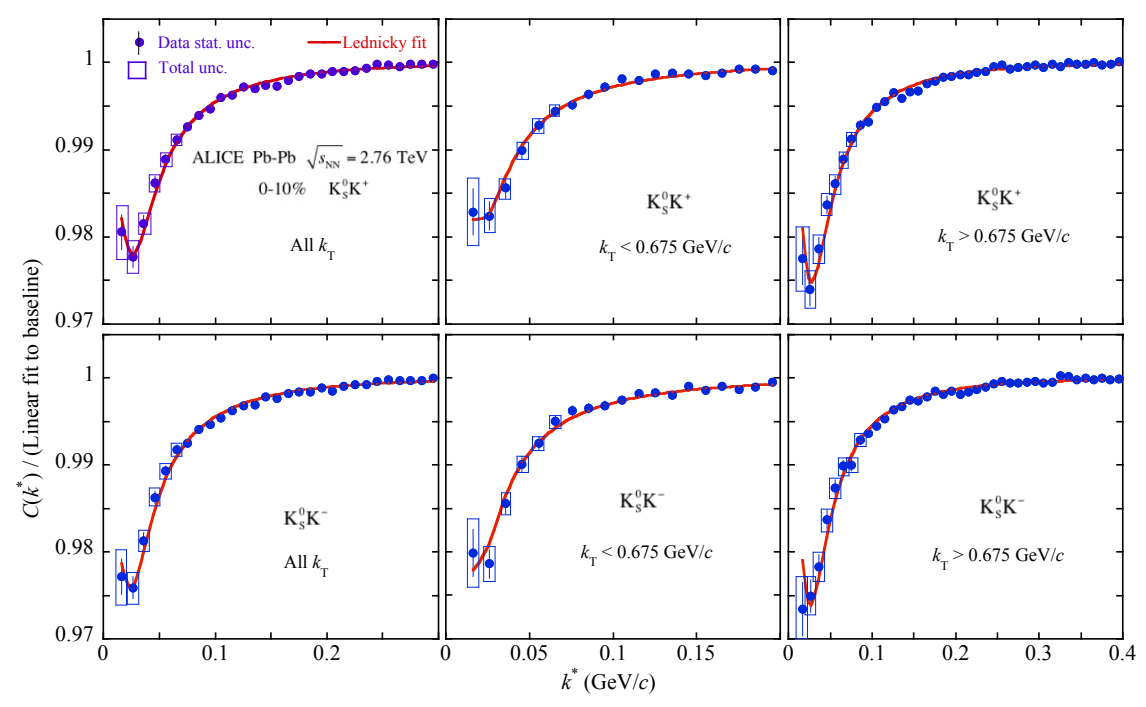

Figure 3. Correlation functions for $\mathrm{K}_{\mathrm{S}}^{0} \mathrm{~K}^{ \pm}$pairs from $\mathrm{Pb}-\mathrm{Pb}$ collisions at $\sqrt{s_{\mathrm{NN}}}=2.76 \mathrm{TeV}$ divided by the linear baseline functions with fits using $\mathrm{a}_{0}$ parameters from [22] ("Achasov2"). Figure from [18]. 


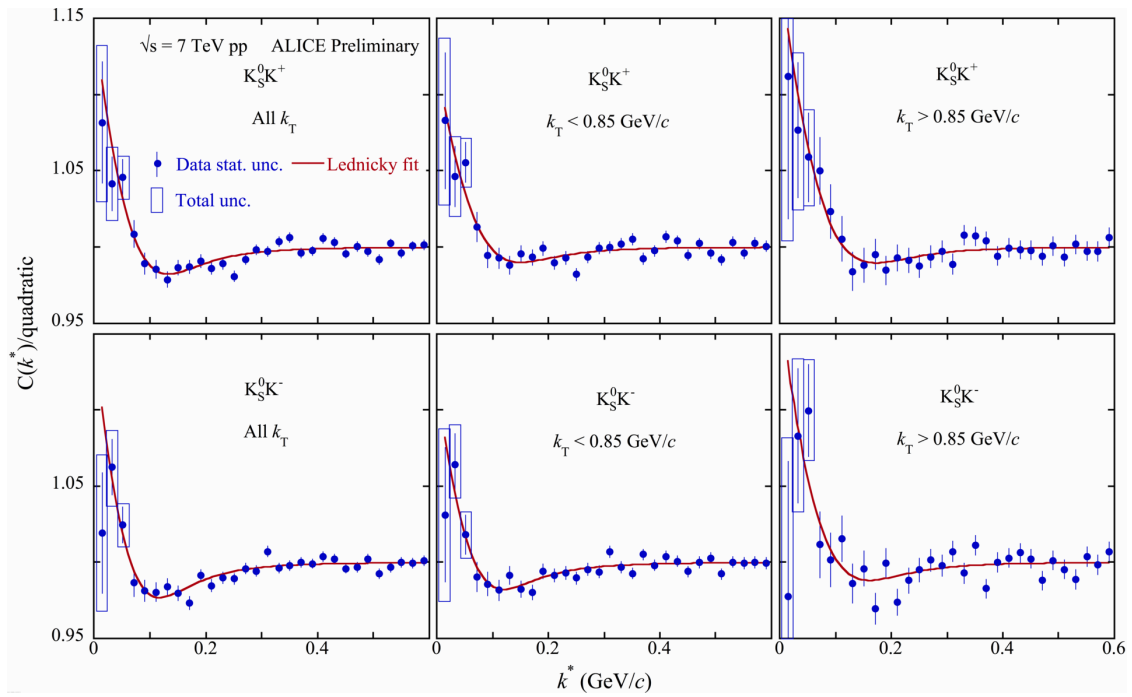

Figure 4. Correlation functions for $\mathrm{K}_{\mathrm{S}}^{0} \mathrm{~K}^{ \pm}$pairs from pp collisions at $\sqrt{s}=7 \mathrm{TeV}$ divided by the quadratic baseline functions with fits using $\mathrm{a}_{0}$ parameters from [22] ("Achasov2").
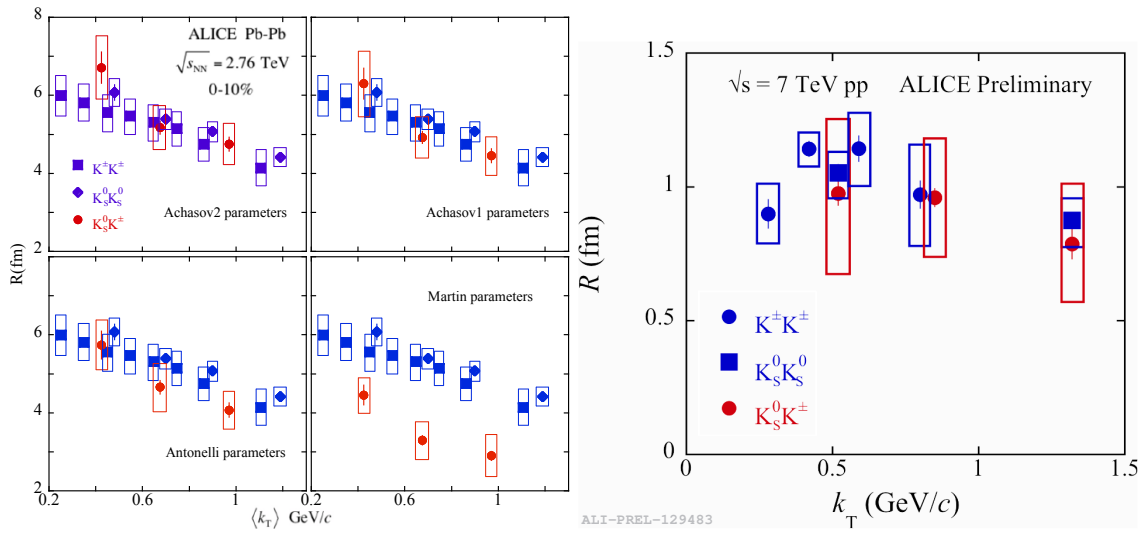

Figure 5. Radius $R$ from $\mathrm{K}_{\mathrm{S}}^{0} \mathrm{~K}^{ \pm}$femtoscopy in $\mathrm{Pb}-\mathrm{Pb}$ collisions at $\sqrt{s_{\mathrm{NN}}}=2.76 \mathrm{TeV}$ (left, plots from [18]) and pp collisions at $\sqrt{s}=7 \mathrm{TeV}$ (right) averaged over the results from fits to $\mathrm{K}_{\mathrm{S}}^{0} \mathrm{~K}^{+}$and $\mathrm{K}_{\mathrm{S}}^{0} \mathrm{~K}^{+}$correlation functions in both collision systems. The $\mathrm{a}_{0}$ parameters used in the fit come from [19-22] for $\mathrm{Pb}-\mathrm{Pb}$ data and from [22] (“Achasov2") for pp data. 


\section{Angular correlations}

Two-particle correlations measured as a function of relative pseudorapidity $\Delta \eta=\eta_{1}-\eta_{2}$ and azimuthal angle $\Delta \varphi=\varphi_{1}-\varphi_{2}$, also referred to as $\Delta \eta \Delta \varphi$ correlations, allow the exploration of a wide spectrum of physics phenomena occurring during high-energy particle collisions. In this paper, the most important findings of the angular correlations analysis in pp collisions at $\sqrt{s}=7 \mathrm{TeV}$ [27] are presented.

The correlation analysis was performed separately for particle-particle and particle-anti-particle cases. Four particle species, namely pions, charged kaons, protons, and lambdas, were measured at relatively low transverse momenta (up to $p_{\mathrm{T}}=2.5 \mathrm{GeV} / c$ ) in the central rapidity region $|\eta|<0.8$. Particle purities exceed $99 \%$ for pions and protons, and are around $96 \%$ for kaons and $95 \%$ for lambdas.

The full two-dimensional $\Delta \eta \Delta \varphi$ distributions can be found elsewhere [27]. In this paper, we focus on one-dimensional $\Delta \eta$-integrated distributions which are directly compared to contemporary Monte Carlo models: PYTHIA6.4 Perugia-0 and PYTHIA6.4 Perugia-2011 [28, 29], PYTHIA8 Monash [30, 31], and PHOJET 1.12 [32]. The experimental results and Monte Carlo calculations for identical particle pairs are shown in Fig. 6, for particle-anti-particle pairs in Fig. 7, while the comparison among different baryon pairs is shown in Fig. 8. One can clearly notice from those plots that all correlations functions except the ones for baryon-baryon (anti-baryon-anti-baryon) case have a typical shape with a near-side peak around $\Delta \varphi=0$ and an away-side correlation around $\Delta \varphi \approx \pi$. This difference is especially pronounced when the measured correlation functions to Monte Carlo models are compared - none of them is able to reproduce the experimental result even qualitatively. Studies performed with EPOS-LHC [33] and HERWIG [34] also do not agree with the measurements.

One has to note that studies similar to those with similar findings were performed in $\mathrm{e}^{+} \mathrm{e}^{-}$collisions at the SLAC-PEP accelerator at $\sqrt{s}=29 \mathrm{GeV} / c$ collision energy [35]. The reported results were explained on the basis of local conservation of baryon number, i.e. in a single parton fragmentation. In this scenario, the production of two baryons in a single fragmentation requires the production of two anti-baryons, which in total results in four heavy particles. This is a very unlikely scenario at this collision energy, where the initial parton energy is small with respect to energies at the LHC. This hypothesis was supported by the models which seemed to describe those data at the time. Current results from the LHC however suggest that there should be an additional, yet unknown, mechanism which suppresses the probability of producing two baryons close in phase-space. The ruled-out scenarios include: (I) Fermi-Dirac quantum statistics - effect present for proton-lambda pairs, (II) Coulomb repulsion - lambda hyperons are neutral particles, (III) strong final-state interaction - produces a positive correlation, and (IV) local baryon number conservation - all studied models have this mechanism implemented.

\section{Summary}

The analysis of two-particle correlations, both in relative momentum (femtoscopy) and $\Delta \eta \Delta \varphi$ space (angular correlations), is an interesting topic in high-energy collisions of protons and heavy ions. Reported results show that both techniques are powerful tools which can be used to study a variety of different phenomena. Applications of femtoscopy beyond traditional measurements of the system size allows for the extraction of previously unknown parameters of the strong interaction for certain particle pairs. In this paper, we show the first measurements of $\Lambda \bar{\Lambda}$ interaction parameters obtained together with those for the $\mathrm{p} \bar{\Lambda}$ system as well as the effective baryon-anti-baryon interaction for those pairs which cannot be measured directly. The conclusion which can be drawn from this study is that the interaction between those systems is similar, it includes a significant annihilation channel, and the strong force itself is either repulsive or bound states are created. The femtoscopic analysis of neutral kaons paired with charged kaons allows for a constraint of the parameters of the $\mathrm{a}_{0}$ resonance and are 
(a) $\pi^{+} \pi^{+}+\pi^{-} \pi^{-}$pairs

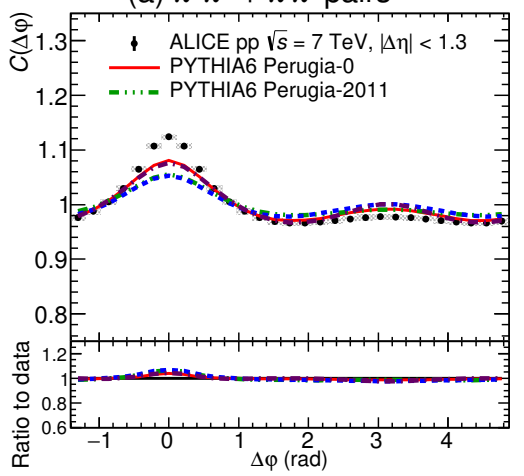

(c) $\mathrm{pp}+\overline{\mathrm{pp}}$ pairs

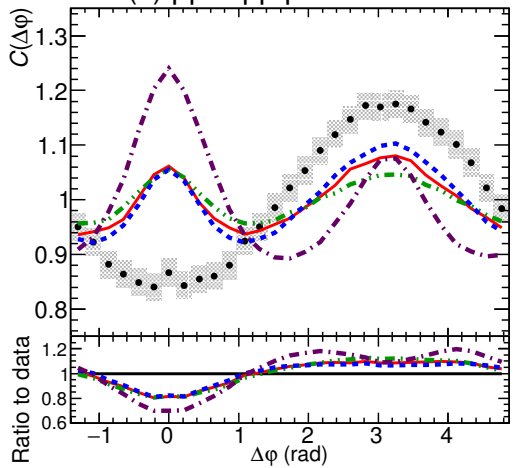

(b) $\mathrm{K}^{+} \mathrm{K}^{+}+\mathrm{K}^{-} \mathrm{K}^{-}$pairs

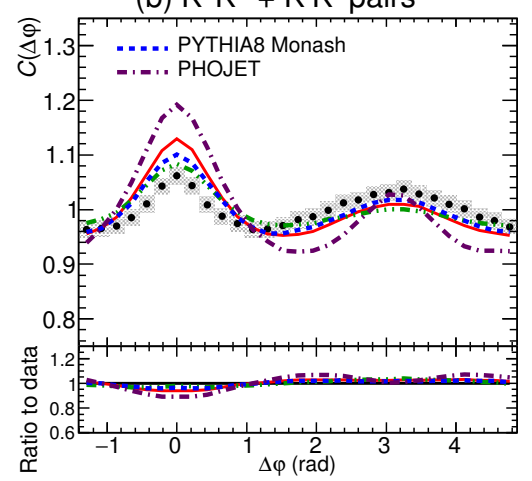

(c) $\Lambda \Lambda+\overline{\Lambda \Lambda}$ pairs

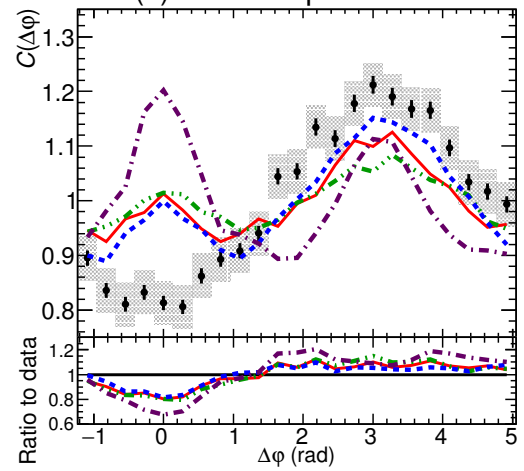

Figure 6. Correlation functions integrated over $\Delta \eta$ for pairs of identical (a) pions, (b) charged kaons, (c) protons, and (d) lambdas from pp collisions at $\sqrt{s}=7 \mathrm{TeV}$ compared to Monte Carlo models. Figure from [27].

compatible with the hypothesis that this particle is indeed a tetraquark state. The angular correlations of baryon-baryon (anti-baryon-anti-baryon) pairs reveal a surprising anti-correlation effect, not explainable by any of currently used Monte Carlo models and the understanding of this result remains an open question.

\section{Acknowledgements}

The author acknowledges support from the Polish National Science Centre grants nos. UMO2015/19/D/ST2/01600, UMO-2014/13/B/ST2/04054, and UMO-2016/22/M/ST2/00176, as well as from the Polish Ministry of Science and Higher Education.

\section{References}

[1] K. Aamodt et al. (ALICE), JINST 3, S08002 (2008)

[2] L. Evans, P. Bryant, JINST 3, S08001 (2008)

[3] E.V. Shuryak, Phys. Lett. B78, 150 (1978), [Yad. Fiz.28,796(1978)] 
(a) $\pi^{+} \pi^{-}$pairs

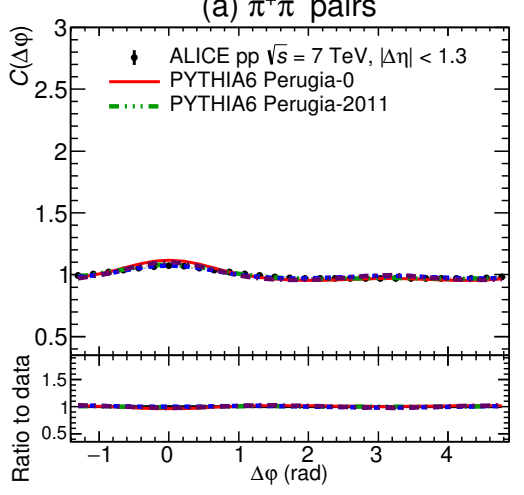

(c) pp pairs

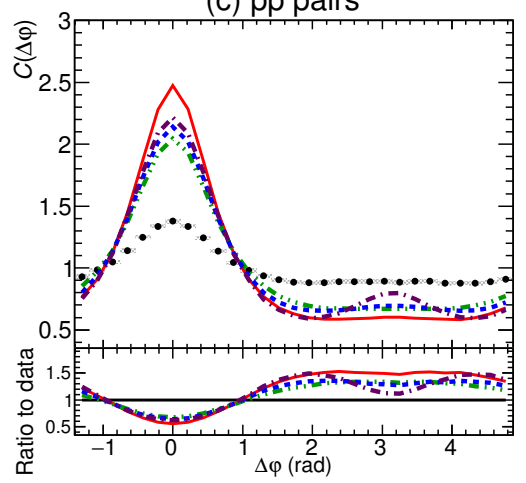

(b) $\mathrm{K}^{+} \mathrm{K}^{-}$pairs

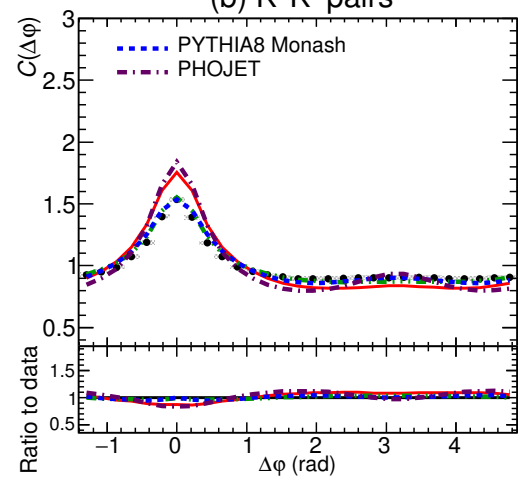

(c) $\Lambda \bar{\Lambda}$ pairs

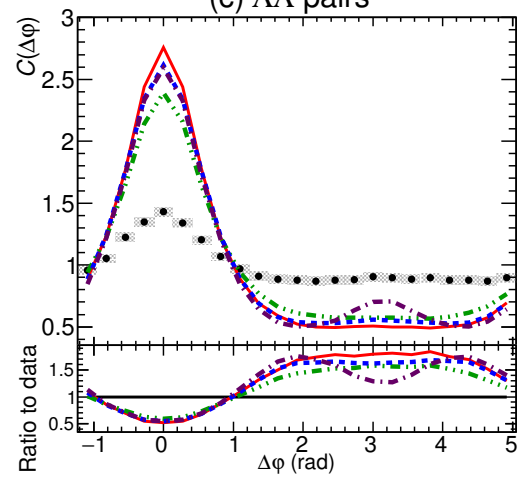

Figure 7. Correlation functions integrated over $\Delta \eta$ for particle-anti-particle pairs of (a) pions, (b) charged kaons, (c) protons, and (d) lambdas from pp collisions at $\sqrt{s}=7 \mathrm{TeV}$ compared to Monte Carlo models. Figure from [27].

[4] J. Adams et al. (STAR), Nucl. Phys. A757, 102 (2005)

[5] K. Adcox et al. (PHENIX), Nucl. Phys. A757, 184 (2005)

[6] B.B. Back et al., Nucl. Phys. A757, 28 (2005)

[7] I. Arsene et al. (BRAHMS), Nucl. Phys. A757, 1 (2005)

[8] S.E. Koonin, Phys. Lett. B70, 43 (1977)

[9] S. Pratt, T. Csorgo, J. Zimanyi, Phys. Rev. C42, 2646 (1990)

[10] R. Lednicky, V.L. Lyuboshits, Sov. J. Nucl. Phys. 35, 770 (1982), [Yad. Fiz.35,1316(1981)]

[11] R. Lednicky, Nucl. Phys. A774, 189 (2006)

[12] C. Patrignani et al. (Particle Data Group), Chin. Phys. C40, 100001 (2016)

[13] M. Bleicher et al., J. Phys. G25, 1859 (1999)

[14] J. Adams et al. (STAR), Phys. Rev. C74, 064906 (2006)

[15] A. Kisiel, H. Zbroszczyk, M. Szymański, Phys. Rev. C89, 054916 (2014)

[16] Z.W. Lin, C.M. Ko, B.A. Li, B. Zhang, S. Pal, Phys. Rev. C72, 064901 (2005) 

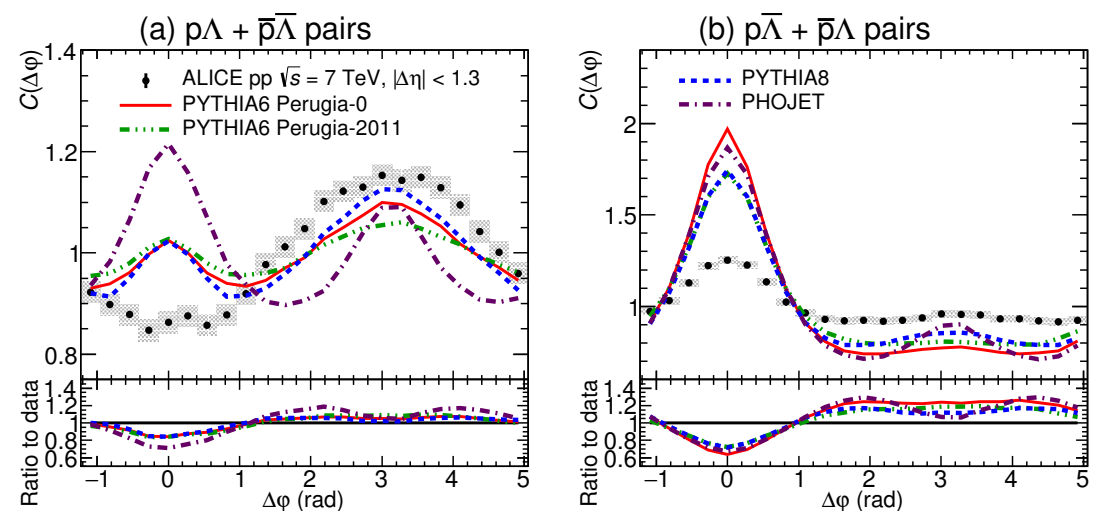

Figure 8. Correlation functions integrated over $\Delta \eta$ for (left) particle-particle and (right) particle-anti-particle correlations of protons with lambdas from pp collisions at $\sqrt{s}=7 \mathrm{TeV}$ compared to Monte Carlo models. Figure from [27].

[17] M. Chojnacki, A. Kisiel, W. Florkowski, W. Broniowski, Comput. Phys. Commun. 183, 746 (2012)

[18] S. Acharya et al. (ALICE), Phys. Lett. B774, 64 (2017), 1705. 04929

[19] A.D. Martin, E.N. Ozmutlu, E.J. Squires, Nucl. Phys. B121, 514 (1977)

[20] A. Antonelli, eConf C020620, THAT06 (2002), [,65(2002)]

[21] N.N. Achasov, V.V. Gubin, Phys. Atom. Nucl. 65, 1528 (2002), [Phys. Rev.D63,094007(2001)]

[22] N.N. Achasov, A.V. Kiselev, Phys. Rev. D68, 014006 (2003)

[23] E. Santopinto, G. Galata, Phys. Rev. C75, 045206 (2007)

[24] R.L. Jaffe, Phys. Rev. D15, 267 (1977)

[25] B. Abelev et al. (ALICE), Phys. Lett. B717, 151 (2012)

[26] B. Abelev et al. (ALICE), Phys. Rev. D87, 052016 (2013)

[27] J. Adam et al. (ALICE), Eur. Phys. J. C77, 569 (2017)

[28] T. Sjostrand, S. Mrenna, P.Z. Skands, JHEP 05, 026 (2006)

[29] P.Z. Skands, Phys. Rev. D82, 074018 (2010)

[30] T. Sjostrand, S. Mrenna, P.Z. Skands, Comput. Phys. Commun. 178, 852 (2008)

[31] P. Skands, S. Carrazza, J. Rojo, Eur. Phys. J. C74, 3024 (2014)

[32] R. Engel, Z. Phys. C66, 203 (1995)

[33] T. Pierog, I. Karpenko, J.M. Katzy, E. Yatsenko, K. Werner, Phys. Rev. C92, 034906 (2015)

[34] G. Corcella, I.G. Knowles, G. Marchesini, S. Moretti, K. Odagiri, P. Richardson, M.H. Seymour, B.R. Webber, JHEP 01, 010 (2001)

[35] H. Aihara et al. (TPC/Two Gamma), Phys. Rev. Lett. 57, 3140 (1986) 